\title{
Astrophysics at X-Ray Spectral Resolution 1000
}

Nancy S. Brickhouse

Harvard-Smithsonian CfA, 60 Garden St., Cambridge, MA 02138

\begin{abstract}
.
The Chandra gratings exceed a resolving power $R=1000$, but only at the longest wavelengths and with relatively low effective areas. Recent X-ray observations of cool stars illustrate the problem of line blending at different resolutions and give a good sense of what we will routinely be able to measure with Constellation-X, provided it has high enough spectral resolution. We also expect new diagnostics from weak lines and line profiles. Support for this work is provided by NASA NAS8-39083 to SAO for the CXC and LTSA NAG5-3559.
\end{abstract}
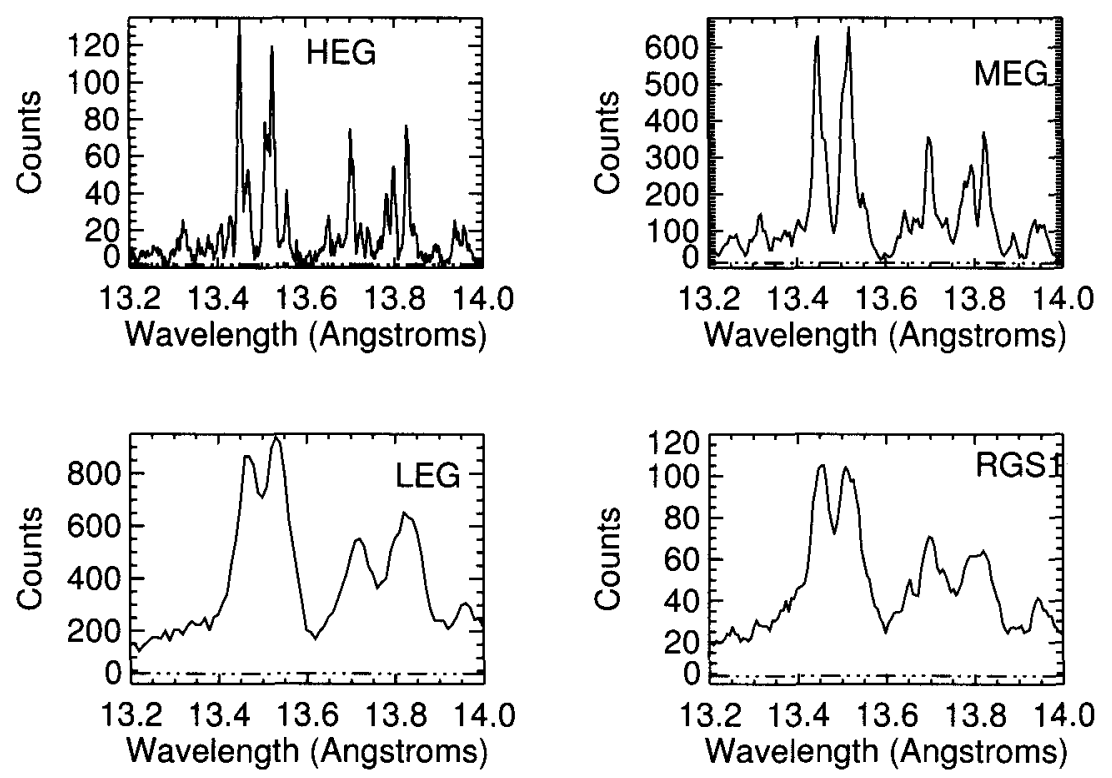

Figure 1. The Ne IX spectral region of Capella at different spectral resolution. At $13.6 \AA$, the $\mathrm{HEG}$ spectrum $(154.7 \mathrm{ks})$ has resolving power $\mathrm{R}=$ $\lambda / \Delta \lambda \sim 1000 ;$ MEG $(154.7 \mathrm{ks})$ has $\mathrm{R} \sim 500$; LEG $(218.5 \mathrm{ks})$ has $\mathrm{R} \sim 270$ and RGS (52.92 ks) has $\mathrm{R} \sim 300$. 


\section{Summary}

Figure 1 shows a comparison of the Ne IX spectral region of Capella observed by Chandra and XMM-Newton (Ness et al. 2003). Although numerous strong lines are observed, the full diagnostic potential, even at $\mathrm{R}=1000$, is not realized because of the modest effective area. Figure 2 shows Lyman series lines, which can provide diagnostics for optical depth and temperature, but require high $\mathrm{S} / \mathrm{N}$. Weak lines such as Lyman series and DR satellite lines provide temperature diagnostics for non-equilibrium ionization plasmas.

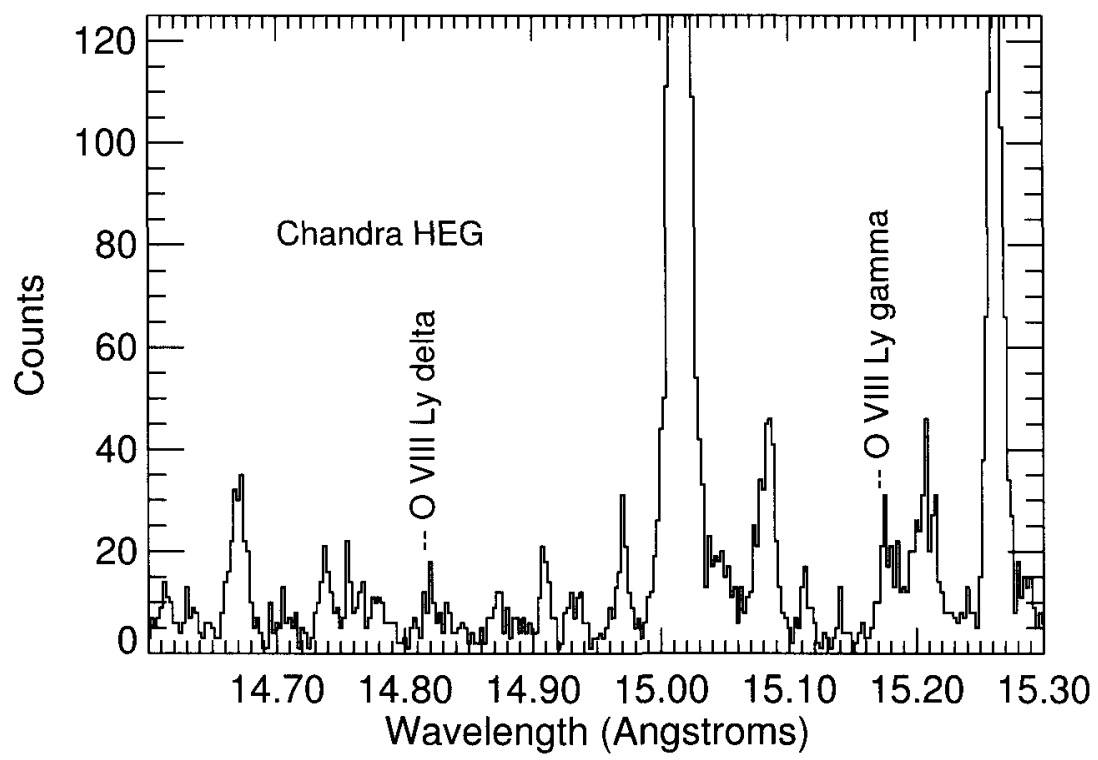

Figure 2. Capella HEG spectrum (154.7 ks) showing two weak O VIII lines. Although $\mathrm{Ly}_{\beta}$ (not shown) is blended with an Fe XVIII line at $\mathrm{R}=1000$, the other lines appear to be relatively unblended from APEC models (Smith et al. 2001) and the observed spectrum.

Line profile diagnostics are accessible with the current gratings, but again with limited $\mathrm{S} / \mathrm{N}$ at the highest resolution. While hot star winds are resolved even at low grating resolving power, motions from cool star coronae can be determined in only a few systems. Brickhouse et al. (2001) measured Doppler shifts $\sim 100 \mathrm{~km} \mathrm{~s}^{-1}$ as a function of phase and determined the locations and sizes of active regions on the contact binary $44 \mathrm{i}$ Boo.

The spectral resolution required for new instrumentation depends on the science case; ultimately, our goal should be to reach the thermal limit $R \sim 10,000$ (Elvis 2001). For R between 3000 and 5000, line profiles for photoionized plasma may be resolved (Fig. 3). The Capella spectrum shows that many diagnostics are blended at $R \sim 1000$, a problem that increases drastically at lower $R$. 


\section{References}

Brickhouse, N. S., Dupree, A. K., \& Young, P. R. 2001, ApJ, 562, L75

Elvis, M. 2001, ASP Conf. Ser. 251, New Century of X-ray Astronomy, 180

Krongold, Y., Nicastro, F., Brickhouse, N. S., Elvis, M., Liedahl, D. A., \& Mathur, S. 2003, ApJ, 597, 832

Ness, J.-U., Brickhouse, N. S., Drake, J. J., \& Huenemoerder, D. P. 2003, ApJ, 598, 1277

Smith, R. K., Brickhouse, N. S., Liedahl, D. A., \& Raymond, J. C. 2001, ApJ, 556, L91
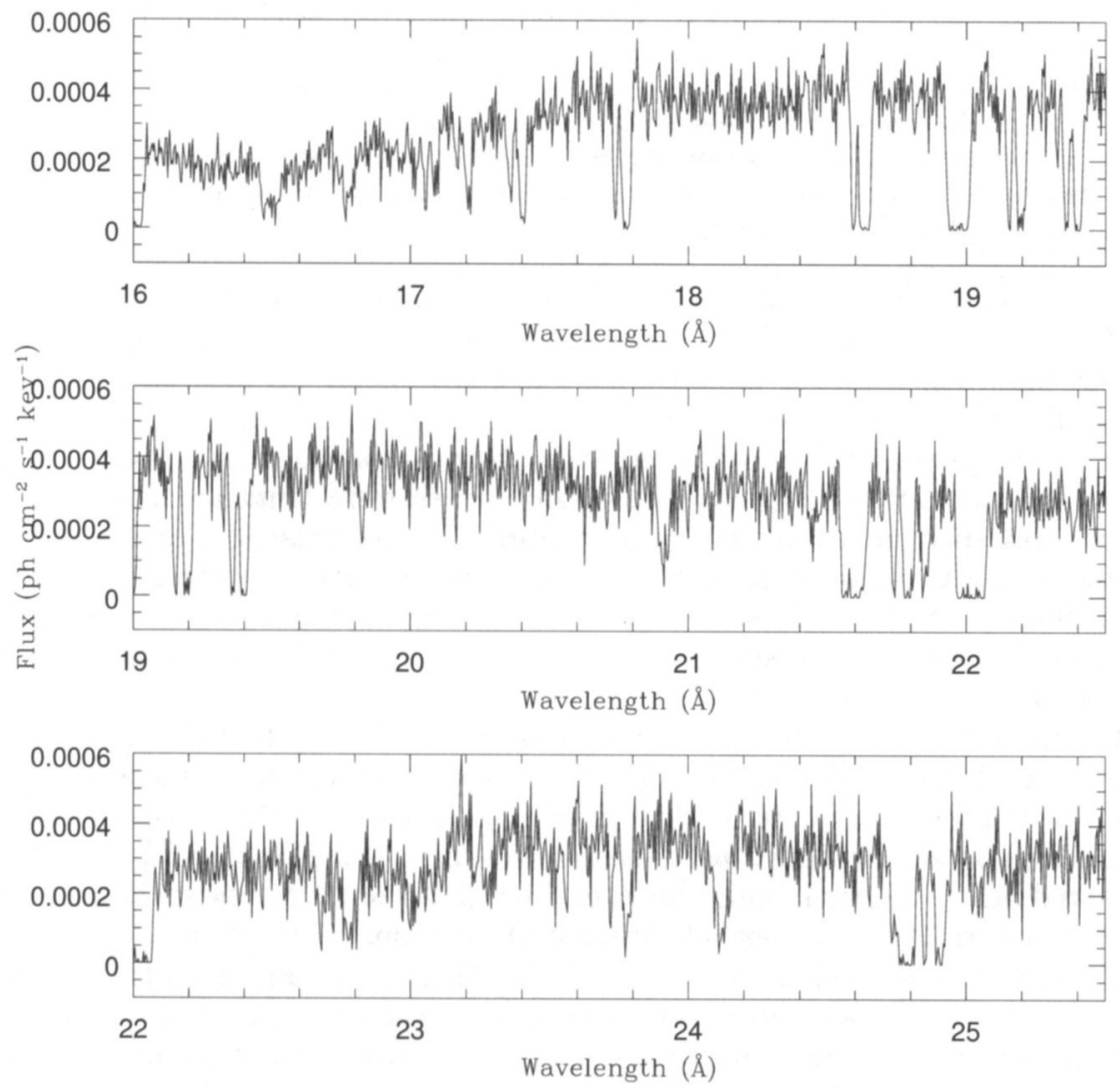

Figure 3. Simulation of NGC 3783 at $\mathrm{R}=5000$, based on Chandra observations (Krongold et al. 2003) with turbulent broadening. Note that one can distinguish between saturated and unsaturated lines at this resolution. Courtesy of Yair Krongold. 